\title{
FRESNEL LAMB WAVE AND V-GROOVE LENSES WITH TUNABLE MODE SELECTIVITY
}

\author{
G.G. Yaralıoğlu, A. Atalar, H. Köymen \\ Electrical and Electronics Engineering Department \\ Bilkent University, 06533 Bilkent, Ankara, TURKEY
}

Abstract - The Lamb wave and V-groove lenses are distinguished by their high surface wave excitation efficiencies. However, due to the fixed incidence angle, a particular lens can only be used for materials having surface wave velocities within a limited range. Hence, it is desirable to have lenses with adjustable incidence angle. Conventional spherical lenses implemented in Fresnel planar lens form have been demonstrated earlier. In this work, Lamb wave and V-groove lenses constructed as Fresnel lenses are presented. We also discuss the feasibility of Fresnel lenses with air as the coupling medium. It is shown that it is possible to build air coupled Fresnel lenses with a reasonable conversion efficiency into subsurface waves.

\section{INTRODUCTION}

The Lamb wave and V-groove lenses are powerful tools for material characterization and SAW velocity measurements $[1,2]$. These lenses insonify the material surface at a fixed incidence angle and large amount of power is converted into the leaky Lamb wave modes. However, if the incidence angle does not match the critical angle for a leaky mode, leaky mode will not propagate along the material surface. For layered materials this difficulty can be overcome by adjusting the excitation frequency and modes can be generated selectively. For solid half spaces it is not possible to generate modes, once the incidence angle fails to match the critical angle. Fresnel version of Lamb wave and V-groove lenses solve the fixed incidence angle problem where the trade off is the degradation of sensitivity. Fresnel Lamb wave lens is composed of concentric conical surfaces such that height and the width of the cones are smaller than the acous-

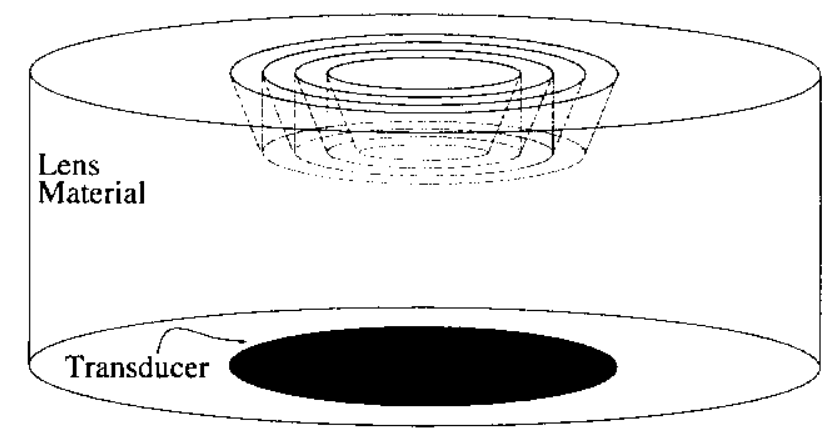

Figure 1: Fresnel Lamb Wave Lens

tic wavelength in the lens material. In the case of Fresnel V-groove lens, the $\mathrm{V}$ shaped groove is replaced by the smaller recesses. Fresnel lenses have planar geometries which make them suitable characterization tool in the air. In the following section, geometry of the lenses are described in detail. Then, Fresnel air transducers with high efficiencies are introduced.

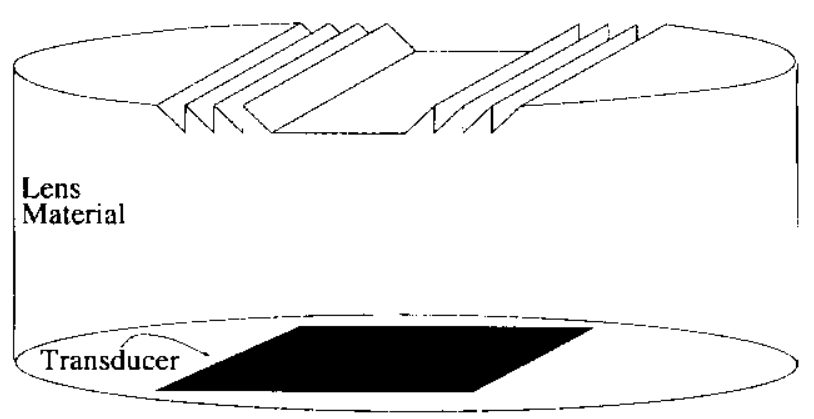

Figure 2: Fresnel V-groove Lens 


\section{FRESNEL LENSES}

The Fresnel acoustic lenses with efficiencies up to $80 \%$ have been reported earlier [3]. There, quadratic surfaces are approximated by a staircase function suitable for silicon micromachining. Fresnel Lamb wave and Fresnel V-groove lenses can be constructed using the same method. Figure 3 shows the cross section of a Fresnel lens.

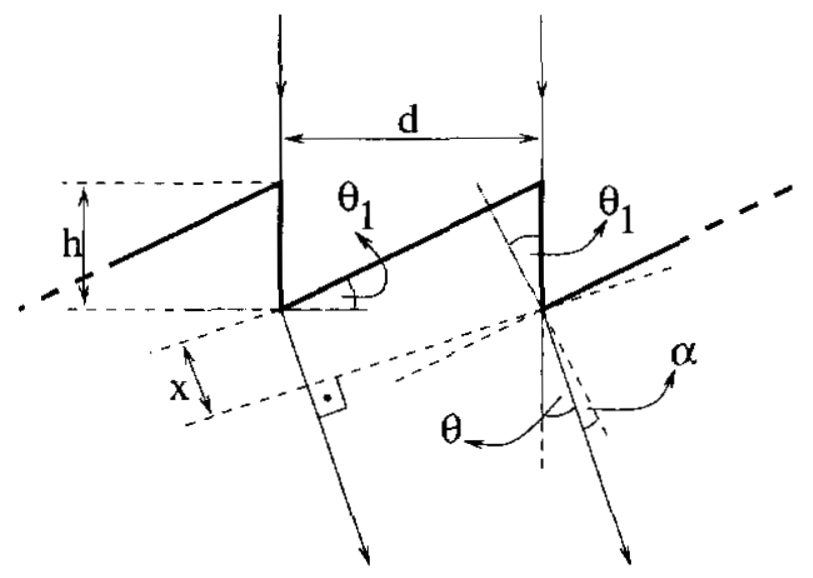

Figure 3: Fresnel lens cross section

Planar geometry limits the band width of the lens. If $f$ designates the operation frequency, minimum allowable pulse width (PW) is

$$
P W=\frac{1}{f} \text { number of grooves } .
$$

Beams refracted at each sawtooth interfere constructively if:

$$
k x=2 \pi,
$$

where $k=2 \pi f / V_{L}$ and $V_{L}$ is the sound velocity in the coupling medium. The distance $x$ is given by

$$
x=\frac{h}{\tan \theta_{1}} \sin \theta,
$$

where $\theta_{1}$ is the wedge angle of the sawtooth, and $\theta$ is the incidence angle. With the substitution (3) into (2), incidence angle, $\theta$, is given

$$
\theta=\arcsin \left(\frac{V_{L} \tan \theta_{1}}{f h}\right) .
$$

We also write a simulation program which calculates field distribution at the lens aperture. The simulation program assumes unity field distribution at the back of the sawtooth like surface of the lens and propagates the field through the refracting element by the ray theory. Fourier transform of aperture field shows the angular spectrum of waves incident on the object surface.

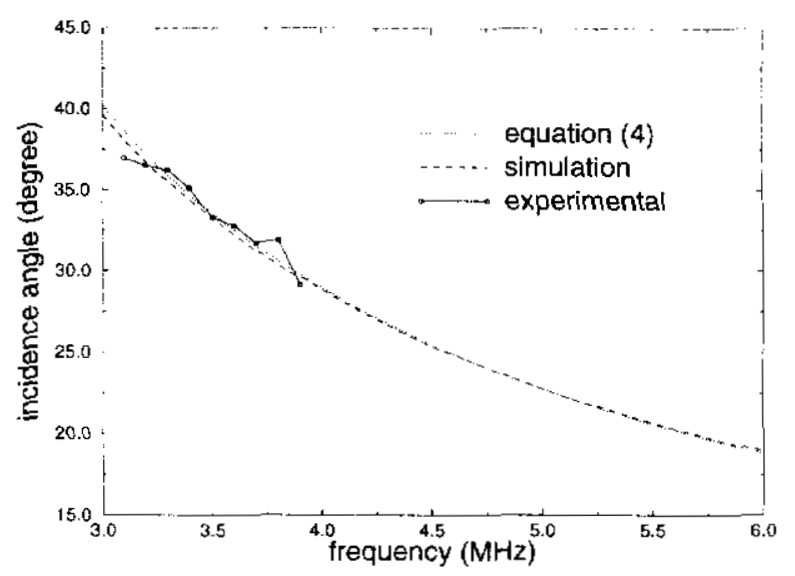

Figure 4: The incidence angle of the acoustic beam refracted by the Fresnel lens.

Figure 4 shows the dependence of the angle to the frequency. The height, $h$, and the angle, $\theta_{1}$, of the sawtooth are chosen as $0.45 \mathrm{~mm}$ and $30^{\circ}$, respectively. Results of the Eq. 4 and simulation match very well. Experimental curve is obtained by calculating the period of the oscillations in $V(z)$ curves from Fresnel Lamb wave lens by employing FFT based algorithm. The lens, with the dimensions indicated in Figure 5, is fabricated from an aluminum block $\left(V_{\text {lens }}=6420 \mathrm{~m} / \mathrm{sec}\right)$ by using a precision lathe. In the experiments a transducer with the center frequency at $3.5 \mathrm{MHz}$ is used and frequency is scanned from $3.1 \mathrm{MHz}$ to $3.9 \mathrm{MHz}$. For this frequency range, incidence angle changes from $29^{\circ}$ to $37^{\circ}$. Material under ob-

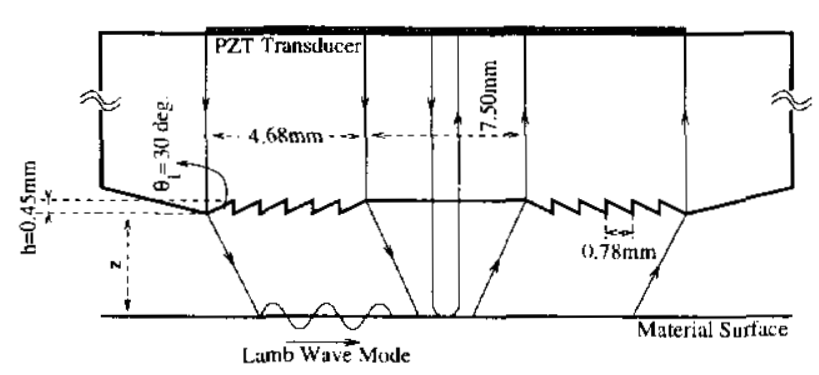

Figure 5: Dimensions of the Fresnel Lamb wave lens used in the experiments. 
servation is brass which has a longitudinal critical at around $20^{\circ}$ and Rayleigh critical angle at around $50^{\circ}$. Hence, with this lens neither longitudinal lateral wave nor Rayleigh wave is excited on the brass sample. So, V(z) oscillations are due to the geometrically reflected beams only and incidence angle is calculated by using the following formula:

$$
\theta=\arccos \left(1-\frac{\lambda_{0}}{2 \Delta z}\right)
$$

where $\lambda_{0}$ is the wavelength in the coupling fluid and $\Delta z$ is the period of the fringes in $V(z)$ curve. Predictions of incidence angle by using theoretical and experimental curves are in agreement.

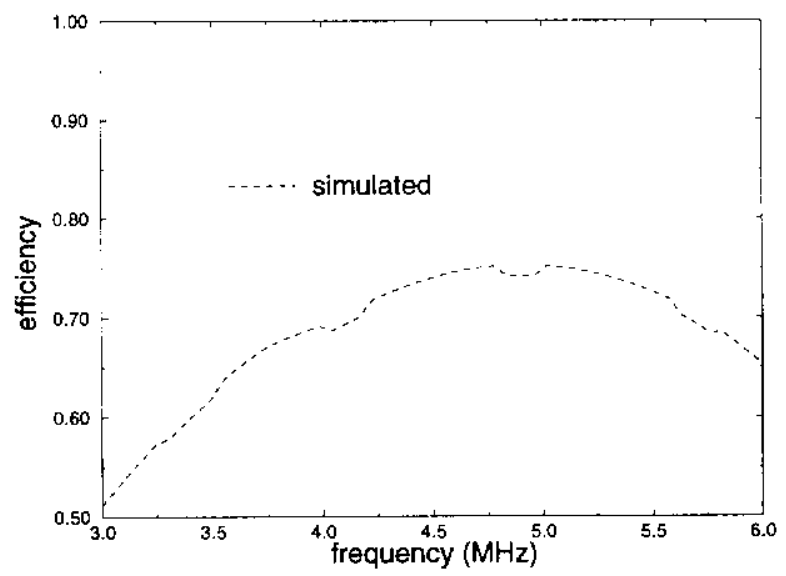

Figure 6: The efficiency of the Fresnel lens.

The efficiency curve (Fig. 6) shows the amount of power sent in the direction of the incidence angle. When frequency is equal to $4.9 \mathrm{MHz}$, incidence angle predicted by Eq. 4 matches with the angle calculated by Snell's law at aluminum/water interface. At this frequency efficiency reaches its maximum value. For frequencies around $4.9 \mathrm{MHz}$ efficiency drops and incidence angle deviates from the value calculated by Snell's law.

The Fresnel lens presented above discussions has a sawtooth like cross section. This geome-

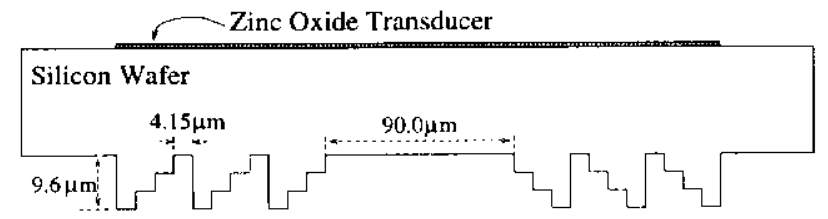

Figure 7: Cross section of high frequency Fresnel Lamb wave or Fresnel V-groove lens. try is suitable for the fabrication of low frequency lenses by using mechanical tools. High frequency lenses can be produced by employing silicon micromachining techniques. In this case sawtooth like cross section of the Fresnel lens is approximated by a stair case function. Figure 7 shows the cross section of silicon micro-machined Fresnel Lamb wave or Fresnel V-groove lens which operates at $200 \mathrm{MHz}$.

The lenses presented to this point use a liquid as coupling medium. It is also possible to produce Fresnel lenses which uses air as the coupling medium. In the next section Fresnel ajr transducers are discussed.

\section{FRESNEL LENSES AS AIR TRANSDUCERS}

Fresnel Lamb wave or Fresnel V-groove lenses can be used as an air transducers. These lenses can be manufactured by silicon micromachining as described in reference [4]. Fig. 8 shows the cross section of a two phase Fresnel air transducer.

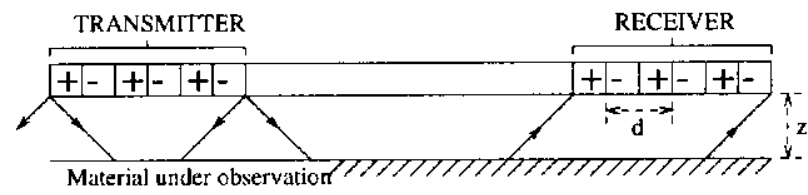

Figure 8: Cross section of an air transducer.

Since geometry of the Fresnel lenses is planar, the distance between lens and the material can be made very small. Although the acoustic attenuation coefficient is very large, sound waves do not attenuate too much while propagating in the air. However, there is a problem at air/solid interfaces. Due to the high impedance mismatches, it is difficult to penetrate into the solids. Table 1 shows typical two way loss values at air/aluminum and air/steel interfaces for excitation of bulk waves.

It is possible to use leaky Lamb wave or Rayleigh modes to increase subsurface excitation efficiency. The optimum transducer size to excite these modes is given by [5]

$$
w=0.6282 \Delta_{s},
$$

where $\Delta_{s}$ is the Schoch displacement and $w$ is the transducer width. For solid/air interfaces Schoch displacement is around $400000 \lambda_{\text {air }}$ which yields 8.6 


\begin{tabular}{l|l|c}
\hline interface & incidence type & two way loss \\
\hline air/aluminum & normal & $80.14 \mathrm{~dB}$ \\
\cline { 2 - 3 } & oblique & $75.07 \mathrm{~dB}$ \\
\hline \multirow{2}{*}{ air/steel } & normal & $88.82 \mathrm{~dB}$ \\
\cline { 2 - 3 } & oblique & $85.26 \mathrm{~dB}$ \\
\hline
\end{tabular}

Table 1: Two way conversion losses for air/solid interfaces.

meter of transducer size at $10 \mathrm{MHz}$. It is, of course, possible to use small transducers with reduced efficiencies. Normalized output voltage of a transducer is calculated by using the following equation $[5]$

$$
V=\frac{\Delta_{s}}{w}\left(1-\exp \left(-\frac{2 w}{\Delta_{s}}\right)\right)^{2} .
$$

By using this formula the two way subsurface excitation efficiency of a $3 \mathrm{~cm}$ wide $10 \mathrm{MHz}$ air transducer for aluminum is calculated as $-41 \mathrm{~dB}$. In Figure 9, a feasible Fresnel Lamb wave air transducer is shown. Air transducer has separate pieces for transmitting and receiving sound waves to avoid receiving specular reflections from the object surface.

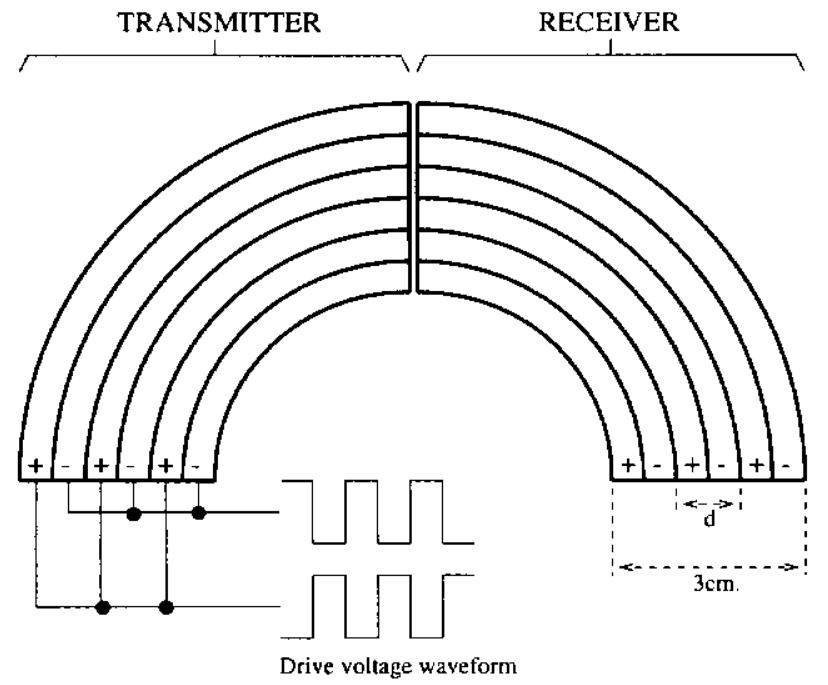

Figure 9: Fresnel Lamb wave air transducer.

The distance $d$ (Fig. 9) is given by:

$$
d=\frac{\lambda_{a i r}}{\sin \theta_{R}} \text {. }
$$

For air/aluminum interface Rayleigh critical angle, $\theta_{R}$, is around $6.6^{\circ}$. Hence, $\mathrm{d}$ is calculated as
$300 \mu \mathrm{m}$ at $10 \mathrm{MHz}$ and minimum allowable pulse width, $(P W)$, is $10 \mu$ sec.

\section{CONCLUSION}

In this study, Fresnel Lamb wave and Fresnel Vgroove lenses are introduced. The main advantage of these lenses is that they release the fixed incidence angle restriction of the Lamb wave and Vgroove lenses. Incidence angle of the Fresnel lenses changes with the excitation frequency. However, their leaky wave excitation efficiencies are lower than that of Lamb Wave and V-groove lenses.

Conventional Lamb wave and V-groove lenses are difficult to fabricate by mechanical means for high frequency applications. On the other hand, Fresnel lenses have planar geometries, hence they can be fabricated on silicon wafer using micromachining techniques. Moreover, it is also possible to build high efficiency air transducers in Fresnel Lamb or V-groove lens forms using methods given in [4] at low $\mathrm{MHz}$ range. With these transducers, much lower two way loss for the excitation of Lamb wave modes can be achieved.

\section{Acknowledgment}

This work is supported by Turkish Scientific and Technical Research Council, TUBITAK.

\section{References}

[1] A. Atalar, H. Köymen, F.L. Deg̃ertekin, "A lamb wave lens for Acoustic Microscopy", IEEE Trans. UFFC, Vol. 39, No. 6, pp. 661667. 1992.

[2] A. Bozkurt, G.G. Yaralığlu, A. Atalar, H. Köymen, "A new directional acoustic lens: Vgroove lens", in IEEE Ultrasonic Proceedings, 1993.

[3] B. Hadimioğlu, E.G. Rawson, R. Lujan R., M. Lim, J.C. Zesch, B.T. Khuri-Yakub, C.F. Quate, "High efficiency Fresnel acoustic lenses", in IEEE Ultrasonic Proceedings, 1993.

[4] M.I. Haller, B.T. Khuri-Yakub, "A surface micro-machined electrostatic ultrasonic air transducer", in IEEE Ultrasonic Proceedings, 1994.

[5] A. Atalar, H. Köymen, "Use of a conical axicon as a surface acoustic wave focusing device", IEEE Trans. UFFC, Vol. 34, No. 1, pp. 53-63, 1987. 